\title{
Effect of endoscopic sinus surgery on irradiation-induced rhinosinusitis in patients with nasopharyngeal carcinoma
}

\author{
Ko-Hsin Hu, MD, Ching-Ting Tan, MD, Kai-Nan Lin, MD, \\ Ya-Jian Cheng, PhD, and Hung-Meng Huang, MD, Taipei, Taiwan
}

\begin{abstract}
OBJECTIVES: We evaluated the effect of endoscopic sinus surgery on irradiation-induced rhinosinusitis of the maxillary-sinus mucosa among patients with nasopharyngeal carcinoma (NPC).

DESIGN: Surgical outcomes were evaluated by changes to the ultrastructure of the antral mucosa and nasomucociliary clearance. METHODS: Twenty-one NPC patients with irradiation-induced chronic sinusitis were enrolled in the study, along with five controls. Specimens were taken from 42 maxillary sinuses during surgery and 1 year after surgery. Saccharin transit time was measured before the initial surgery and 1 year after surgery.

RESULTS: In the postoperative cases, we found a decrease in the number of the submucosal gland openings $(P<0.05)$, the cilia in the antral mucosa regenerated $(P<0.05)$, and the saccharin transit time reduced $(P<0.05)$; the number of goblet cells did not change.

CONCLUSION: Endoscopic sinus surgery is an effective treatment for irradiation-induced rhinosinusitis in NPC patients, improving ventilation and drainage of the paranasal sinuses, and facilitating regeneration of the sinus mucosa.

(C) 2008 American Academy of Otolaryngology-Head and Neck Surgery Foundation. All rights reserved.
\end{abstract}

Jasopharyngeal carcinoma (NPC) is more prevalent in 1 Taiwan than in Western countries. ${ }^{1}$ The mainstay of treatment for NPC is radiotherapy, which is usually associated with mucosal side effects at the tumor site and its surrounding structures. ${ }^{1}$ Sinusitis is one of these side effects and is very bothersome to NPC patients after irradiation. New treatments that relieve patient discomfort and help improve quality of life are urgently needed.

Most previous studies of the sequelae of radiation therapy for treatment of NPC mainly focused on the relationships between radiotherapy and mucosal change in the middle ear. ${ }^{2-4}$ Nonetheless, the radiation treatment for NPC is also associated with impairment of nasomucociliary clearance (NMCC) in the paranasal sinuses. ${ }^{5,6}$ Comparatively few studies focused on the effects of radiotherapy on the paranasal sinuses. ${ }^{5-7}$

Functional endoscopic sinus surgery (FESS) is widely used to treat various lesions of the osteomeatal complex in patients with chronic sinusitis. ${ }^{8}$ Because FESS procedures elicit minimal injury to the surrounding sinus tissue, this treatment modality offers the possibility of successful adaptation to the treatment of chronic sinusitis in NPC patients after radiotherapy.

The aim of this study was to evaluate the effect of FESS on NPC patients with radiotherapy-induced chronic rhinosinusitis. The effects of FESS on any pathologic changes to the maxillary-sinus mucosa occurring during a 1-year period after FESS were analyzed.

\section{MATERIALS AND METHODS}

From January 2001 to December 2006 inclusively, 21 NPC patients ( 8 women and 13 men) who underwent previous radiotherapy with subsequent chronic rhinosinusitis were enrolled in the prospective study. The age of the patients ranged from 43 to 58 years (mean, 49.5 years). The average irradiation dose to the nasopharynx was $74 \mathrm{~Gy}$ (range, 70 to $80 \mathrm{~Gy}$ ) and the mean interval between irradiation and FESS was 2.1 years (range, 1.2 to 4.0 years). NPC patients with nasal polyps, a history of sinonasal surgery, or symptoms and sinus tomographic findings related to sinusitis before receiving radiation therapy were excluded.

All patients exhibited signs and symptoms of chronic paranasal sinusitis such as presence of yellowish rhinorrhea, nasal obstruction, postnasal drip, and hyposmia that had not responded to antibiotic treatment in the preceding 3 months. Each patient underwent computed tomography (CT) of the sinuses that documented sinus opacification and correlated with clinical findings. The criteria of American Academy of Otolaryngology-Head and Neck Surgery task force (2003) were used to define CRS in the study. ${ }^{9}$ The preoperative CT images revealed that all 42 maxillary sinuses (21 patients) were affected, followed by the anterior ethmoid sinuses $(25$ of 42 sides) and ostiomeatal complexes ( 25 of 42 sides) and 2008.

Received March 18, 2008; revised June 13, 2008; accepted July 7, 
posterior ethmoid sinuses ( 8 of 42 sides). None of the frontal sinuses or sphenoid sinuses was affected.

Choanal stenosis was found in five of the 21 patients before surgery. Four of the five patients had unilateral choanal stenosis and one of the five patients had bilateral choanal stenosis. Six of the 21 patients had nasal synechia. Four patients had unilateral nasal synechia and two of the six patients had bilateral nasal synechia. Choanal stenosis and nasal synechia were surgically treated on the completion of FESS. The saccharin transit time measured in the nasal cavity with choanal stenosis (6 of 42 sides) and nasal synechia ( 8 of 42 sides) were removed from the STT comparison data to avoid introducing significant confounding variables that affect the interpretation of the results of FESS. All 21 patients were instructed to begin nasal irrigation with normal saline solution with a bulb syringe twice a day. Endoscopic nasal cleaning was performed once every 2 weeks.

Each patient's bio-data with respect to irradiation dose and duration of sinusitis subsequent to radiotherapy was obtained at the time of enrollment at Taipei City Hospital. Patients underwent endoscopic nasal cleaning each month after surgery. All patients underwent endoscopic sinus surgery with the use of the techniques described in 1985 by Kennedy et al. ${ }^{8,10}$ After uncinectomy, a middle meatal antrostomy was performed. The antral mucosa remained mostly intact and any exudates were cleaned from the antrum only.

Whether surgeons performed posterior ethmoidectomy, frontal-sinus clearance, or sphenoidotomy or not, depended on the relative extent of sinus disease. Biopsy specimens were taken from the superolateral wall of the maxillary sinus during surgery and also at follow-up 1 year postoperatively. In addition, 10 specimens of normal antral mucosa were acquired by limited maxillary antrostomy with partial removal of the posterior frontanell from five patients who underwent septomeatoplasty for relieving chronic nasal obstruction due to deviated nasal septum and hypertrophic inferior turbinates and served as the control group. Informed consent approved by the Bioethical Committee of the hospital was obtained from patients before enrollment in the study.

To investigate the effect of FESS on the maxillary sinus mucosa of patients, specimens from the superolateral wall of the antrum were collected during surgery and at the 1-year postoperative follow-up visit. Specimens were washed in saline solution to remove surface blood and mucus. Preparation for scanning electron microscopy (SEM) included prefixation with 2.5 percent glutaraldehyde, sequential dehydration in graded ethanol solutions $(30 \%, 50 \%, 70 \%$, $80 \%, 90 \%, 95 \%$, and $100 \%$ ), critical-point drying with carbon dioxide, and then sputter coating with gold. All specimens were observed with an SEM (S-2500, Hitachi, Tokyo, Japan) in the Institute of Zoology, Academia Sinica, Taipei.

Goblet cells and submucosal glands openings were counted in five randomly chosen visual fields by SEM $(\times 2400)$ and the average of the counts of five visual fields was taken to represent each specimen.

The ciliary area (CA) was defined as the area occupied by the cilia on the epithelial surface of the mucosal specimen taken from the superolateral wall of the maxillary sinus. To determine the CA, the photographic images of each sinus specimen observed under the SEM $(\times 700)$ was scanned into the computer, and five randomly chosen visual fields occupied by cilia on the screen were then manually circumscribed. A software program (Image Pro, version 3) was used to calculate the ciliary area and the average CA of five visual fields was taken to represent each specimen. Student's $t$ tests with 95 percent confidence intervals were used to compare the results for each group.

A saccharin test was used to determine the NMCC. ${ }^{11}$ With the patient in a sitting position, a saccharin granule was placed on the septal mucosa corresponding to the anterior-inferior tip of the middle turbinate. The time required for the patient to experience a sweet taste was measured. When, after a period of 60 minutes, a patient failed to experience a sweet taste, the result was recorded as a score of greater than 60 minutes and treated as a 60-minute score in the statistical analysis. Because choanal stenosis and nasal synechia are significant confounders, the saccharin transit time measured in the nasal cavity with choanal stenosis (6 of 42 sides) and nasal synechia ( 8 of 42 sides) were removed from the STT comparison data. The mean saccharin transit time of 10 normal adults ( 20 sides), different from the control group who underwent biopsy of antral mucosa, was taken as the control.

Student's $t$ tests with 95 percent confidence intervals were used to compare the pre- and postoperative saccharin test results.

\section{RESULTS}

For the control group, the mean number of goblet cells and submucosal-gland openings per visual field were $3.70 \pm$ 0.82 and $4.20 \pm 1.03$, respectively (Table 1 ). In the FESS group, the intraoperative specimens had a similar mean number of goblet cells $(3.64 \pm 0.93)$, but a significantly higher mean number of submucosal-gland openings per visual field $(6.86 \pm 1.46)$, compared to their postoperative analog (3.60 \pm 0.86 and $5.02 \pm 1.33$, respectively). For the antral mucosa, the number of goblet cells per visual field in the intraoperative group was not significantly less than in the control group $(P>0.05)$. The number of goblet cells per visual field of post-FESS antral mucosa at the 1-year postoperative visit was not significantly less than that in the intraoperative and control groups $(P>0.05$, for both). The number of submucosal gland openings in the antral mucosa significantly decreased after FESS $(P<0.01)$, but remained significantly greater than in the control group $(P<0.05)$ (Table 1).

The CA of normal controls was $93.60 \pm 4.74$ percent. By comparison, the $\mathrm{CA}$ of the maxillary mucosa from the 
Table 1

Study group

\begin{tabular}{lcccc}
\hline & $\begin{array}{c}\text { Goblet cells } \\
\text { (per vf) } \\
\text { Mean }(95 \% \mathrm{Cl})\end{array}$ & $\begin{array}{c}\text { Submucosal gland } \\
\text { openings (per vf) } \\
\text { Mean }(95 \% \mathrm{Cl})\end{array}$ & $\begin{array}{c}\text { Ciliary area (\%) } \\
\text { Mean (95\% Cl) }\end{array}$ & $\begin{array}{c}\text { Saccharin transit } \\
\text { time (minute) } \\
\text { Mean }(95 \% \mathrm{Cl})\end{array}$ \\
\hline Intraoperative & $3.64^{\mathrm{a}}(3.36 \sim 3.92)$ & $6.86^{\mathrm{d}}(6.42 \sim 7.30)$ & $29.50^{\mathrm{f}}(24.68 \sim 34.33)$ & $44.32^{\mathrm{h}}(38.43 \sim 50.21)$ \\
Postoperative & $3.60^{\mathrm{b}}(3.32 \sim 3.86)$ & $5.02^{\mathrm{e}}(4.62 \sim 5.43)$ & $67.21^{\mathrm{g}}(60.52 \sim 73.91)$ & $28.86^{\mathrm{i}}(24.02 \sim 33.67)$ \\
Control & $3.70^{\mathrm{c}}(3.19 \sim 4.21)$ & $4.20(3.56 \sim 4.84)$ & $93.60(90.66 \sim 96.54)$ & $14.70(12.47 \sim 16.93)$ \\
\hline
\end{tabular}

$\mathrm{Cl}$, Confidence interval; vf, visual field.

$\mathrm{N}=42$ for submucosal gland openings, goblet cells, ciliary area, and saccharin transit time for the antral mucosa of previously irradiated NPC patients during and following FESS for submucosal gland openings, goblet cells, and ciliary area, respectively; N = 28 for saccharin transit time. Control group: $\mathrm{N}=10$ for submucosal gland openings, goblet cells, and ciliary area, respectively; $\mathrm{N}=20$ for saccharin transit time.

$P<0.05: \mathrm{d}$ vs e, f vs $\mathrm{g}$, h vs i.

$P>0.05:$ a vs b.

superolateral-wall specimens collected during surgery was $29.50 \pm 15.96$ percent (Fig 1), and for the postoperative specimens was $67.21 \pm 22.14$ percent (Fig 2). The mean postoperative $\mathrm{CA}$ was significantly greater than the preoperative value $(P<0.05)$.

The saccharin transit time for the normal controls of $14.70 \pm 5.08$ minutes was significantly shorter than the preoperative and the postoperative ST for the patients treated with FESS $(44.32 \pm 15.91$ and $28.86 \pm 13.00$ minutes, respectively) (Table 1). The saccharin transit time decreased significantly 1 year postoperatively, compared with the intraoperative analog $(P<0.05)$.

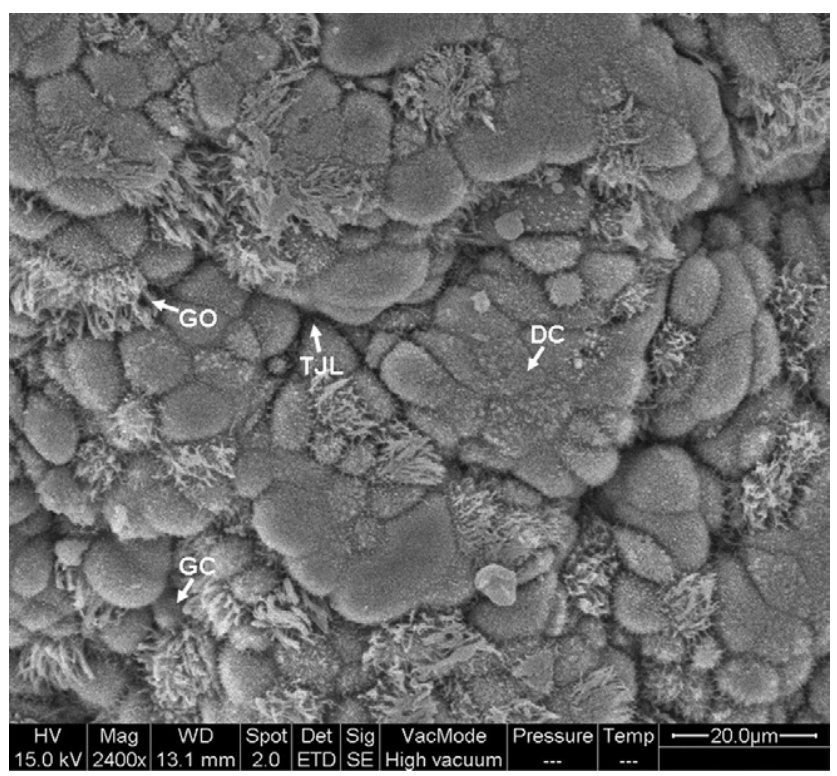

Figure 1 Scanning electron microscopic image of a maxillarysinus mucosa specimen from a 46-year-old woman harvested intraoperatively shows numerous de-ciliated cells $(D C)$. Note also some submucosal gland openings $(G O)$, goblet cells $(G C)$, and the loosening of the tight junctions (TJL).

\section{DISCUSSION}

The NMCC of the nasal cavity and paranasal sinuses depends on proper functioning of the mucosal cilia and the various properties of the mucus present, and finally, their proper interactions. ${ }^{12}$ A variety of factors, including infection, dry air, and irradiation, impair NMCC. ${ }^{13,14}$ Animal experiments also demonstrated that irradiation of the respiratory mucosa damage mucosal cilia. ${ }^{15}$ Ohashi et al ${ }^{16}$ reported an increase in goblet-cell secretion, vacuolation of the ciliated cells, and decreased ciliary activities of rabbit mucosa in the first 4 weeks after irradiation. Delayed effects of irradiation on the respiratory mucosa reportedly occur in conjunction with the transformation of epithelial cells into a stratified arrangement, with a reduction of cytoplasmic vol-

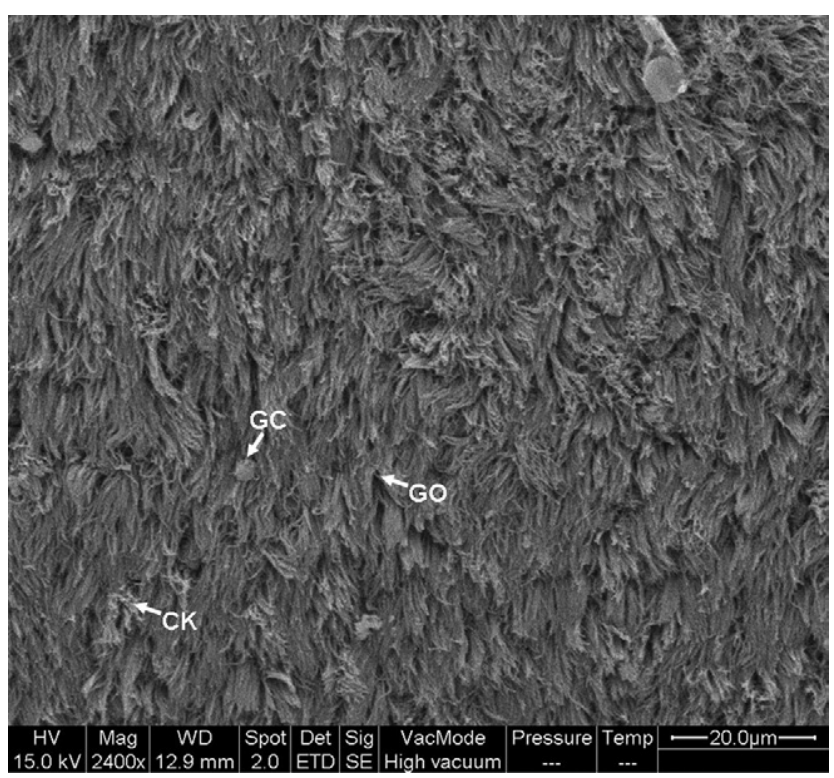

Figure 2 Scanning electron microscope image of the maxillary sinus mucosal tissue of a 46-year-old woman harvested 1 year after FESS shows full mucosal recovery, with ciliated cells, some gland openings $(G O)$, and goblet cells $(G C)$. Note ciliary knobs $(C K)$. 
ume. Ultrastructural studies revealed areas of ciliary loss, intercellular and intracellular vacuolation, and ciliary dysmorphism. ${ }^{2,5}$ Although irradiation-induced ciliary dysmorphism may compromise the NMCC of the sinuses and result in sinusitis, chronic sinusitis itself may also induce the formation of dysmorphic cilia, which subsequently exacerbates the already hampered NMCC. They interact reciprocally to become a vicious cycle in the pathogenesis of irradiation-induced sinusitis.

FESS is an effective method for treating patients with certain medically recalcitrant diseases, including sinusitis in patients with primary ciliary dyskinesia. ${ }^{17}$ Therefore, we performed FESS on NPC patients with irradiation-induced chronic sinusitis for symptomatic improvement. Parsons and Greene ${ }^{17}$ reported that FESS in three children with primary ciliary dyskinesia led to remarkable improvement in their presurgical level of discomfort. This outcome suggests that FESS results in symptomatic improvement in these patients by improving the ventilation and drainage of the sinuses.

Elwany $^{2}$ reported that irradiation significantly increased the number of submucosal glands present and nonsignificantly decreased the number of goblet cells in six patients with otitis media at 4 to 11 months after treatment. Si et al ${ }^{6}$ reported that radiotherapy treatment decreased the number of goblet cells and increased the number of the submucosal glands in the ethmoid sinus mucosa 1 to 3 years after therapy in a series of 15 NPC patients. This led to the development of symptoms of rhinosinusitis in the 15 patients. We found no reports on the effect of FESS on the ultrastructure of the sinus mucosa in NPC patients with postirradiation rhinosinusitis. We found that the number of goblet cells in the maxillary sinus mucosa was lower in the intraoperative specimens from NPC patients with irradiation-induced chronic rhinosinusitis than in the normal control group of patients who underwent septomeatoplasty $(P>0.05)$. The number of submucosal gland openings in the antral mucosa significantly decreased after FESS $(P<$ $0.01)$, but remained significantly greater than in the control group $(P<0.05)$. The increase in the number of the submucosal glands after radiotherapy could indicate new gland formation $^{2}$; whether the gland activity is normal or not warrants further investigation.

The cilial surface density is very high in all parts of the normal maxillary sinus (range, $91.3 \%$ to $97.7 \%$ ). ${ }^{18}$ Lou et $\mathrm{al}^{5}$ used ultrastructural studies to investigate ciliary loss at the infundibular epithelium and found areas of ciliary loss, intercellular and intracellular vacuolation, and ciliary dysmorphism an average of 5.9 years after radiotherapy. Bassiouny et $\mathrm{a}^{19}$ reported that patients with chronic paranasal sinusitis had cilial surface densities in the superolateral maxillary sinus walls that were 10.12 percent of normal levels intraoperatively, and increased to 50.72 percent 8.1 months after FESS. We found that the CA value was 28.62 percent of the normal control intraoperatively and recovered to 66.70 percent one year after FESS. Although it is still possible that chronic rhinosinusitis and not irradiation is the cause for this finding, nonetheless, this finding suggests that even in NPC patients with postirradiation chronic rhinosinusitis, the potential for epithelial recovery still exists after FESS and is similar to the epithelial recovery of sinus mucosa in patients with chronic sinusitis after FESS.

The saccharin test is a simple, inexpensive, and easy method to measure the NMCC of the sinus mucosa. Canciani et $\mathrm{al}^{20}$ demonstrated that patients with suspected primary ciliary dyskinesia who had saccharin test results in the range of 30 to 60 minutes had some degree of ciliary abnormality that manifested as a persistence of lower ciliary motility or at best, a relatively normal frequency of cilial beat. We found that the saccharin transit time recorded immediately before FESS significantly improved by 1 year after FESS. This finding indicates that the NMCC of the sinus mucosa improved after FESS in NPC patients with irradiation-induced chronic rhinosinusitis as a consequence of better ventilation and drainage.

Based on our data, FESS could effectively relieve secondary sinusitis induced by the irradiation of the mucosa, and thus facilitate the recovery of the ultrastructure of the sinus mucosa. However, further research with the postoperative follow-up longer than 1 year would be needed to determine the effect of FESS on the antral mucosa because radiotherapy may cause long-term damage to the sinonasal mucosa. ${ }^{5}$

\section{CONCLUSION}

We showed that FESS is effective for the treatment of irradiation-induced rhinosinusitis in NPC patients through the improvement of the ventilation and drainage of the paranasal sinuses. Nonetheless, a further prospective research in a larger group of patients with the postoperative follow-up more than 1 year longer would be needed to determine the effect of FESS on the postirradiated sinus mucosa.

\section{ACKNOWLEDGEMENT}

The authors express their gratitude to Mr Pung-Pung Hwang, the Deputy Director of the Institute of Zoology of Academia Sinica in Taiwan, who provided substantial technical and material support for this study.

\section{AUTHOR INFORMATION}

From the Department of Otorhinolaryngology-Head and Neck Surgery (Dr $\mathrm{Hu}$, Keelung Hospital, Department of Health; the Department of Otolaryngology (Drs Tan and Lin), National Taiwan University Hospital and National Taiwan University College of Medicine, Taipei; the Department of Life Science (Dr Cheng), National Taiwan University, Taipei; and the Department of Otorhinolaryngology (Dr Huang), Taipei City Hospital, Taipei Medical University, Taipei, Taiwan. 
Corresponding author: Dr Hung-Meng Huang, Department of Otorhinolaryngology, Taipei City Hospital, Taipei Medical University, 12 Fu Chou Street, Taipei, Taiwan.

E-mail address: hmengh@yahoo.com.tw.

\section{AUTHOR CONTRIBUTION}

Ko-Hsin Hu, data acquisition, manuscript revision; Ching-Ting Tan, manuscript draft; Kai-Nan Lin, data analysis and interpretation; Ya-Jian Cheng, statistical analysis; Hung-Meng Huang, study concept and design, study supervision, manuscript revision.

\section{FINANCIAL DISCLOSURE}

None.

\section{REFERENCES}

1. Hsu MM, Tu SM. Nasopharyngeal carcinoma in Taiwan: clinical manifestations and results of therapy. Cancer 1983;52:362-8.

2. Elwany S. Delayed ultrastructural radiation induced changes in the human mesotympanic middle ear mucosa. J Laryngol Otol 1985;99: $343-53$.

3. Ohashi Y, Nakai Y, Esaki Y, et al. An experimental study of the mucociliary pathology of the eustachian tube in otitis media with effusion after irradiation. Arch Otolaryngol Head Neck Surg 1989; 246:428-32.

4. Ohashi Y, Nakai Y, Esaki Y, et al. Acute effects of irradiation on middle ear mucosa. Ann Otol Rhinol Laryngol 1988;97:173-8.

5. Lou PJ, Chen WP, Tai CC. Delayed irradiation effects on nasal epithelium in patients with nasopharyngeal carcinoma. Ann Otol Rhinol Laryngol 1999;108:474-80.

6. Si Y, Wei H, Huang B, et al. Histomorphological changes of ethmoid sinus mucosa in sinusitis patients with nasopharyngeal carcinoma after radiotherapy. Chin Arch Otolaryngol Head Neck Surg 2005;12:729-32.
7. Porter MJ, Leung SF, Ambrose R, et al. The paranasal sinuses before and after radiotherapy for nasopharyngeal carcinoma: a computed tomographic study. J Laryngol Otol 1996;110:19-22.

8. Kennedy DW, Zinreich SI, Rosenbaum AE, et al. Functional endoscopic sinus surgery. Theory and diagnostic evaluation. Arch Otolaryngol 1985;111:577-82.

9. Benninger MS, Ferguson BJ, Hadley JA, et al. Adult chronic rhinosinusitis: definitions, diagnosis, epidemiology, and pathophysiology. Otolaryngol Head Neck Surg 2003;129:S1-32.

10. Kennedy DW. Functional endoscopic sinus surgery: technique. Arch Otolaryngol 1985;111:643-9.

11. Sakakura Y, Majima Y, Harada T, et al. Nasal mucociliary transport of chronic sinusitis in children. Arch Otolaryngol Head Neck Surg 1992; 118:1234-7.

12. Sakakura Y, Majima Y, Saida S, et al. Reversibility of reduced mucociliary clearance in chronic sinusitis. Clin Otolaryngol 1985; 10:79-83.

13. Littlejohn MC, Stiernberg CM, Hokanson JA, et al. The relationship between the nasal cycle and mucociliary clearance. Laryngoscope 1992;102:117-20.

14. Stringer S, Stiles W, Slattery WH 3rd, et al. Nasal mucociliary clearance after radiation therapy. Laryngoscope 1995;105:380-2.

15. Fujiwara K, Hakansson $\mathrm{CH}$, Toremalm NG. Influence of ionizing radiation on ciliary cell activity in the respiratory tract. Acta Radiol Ther Phys Biol 1972;11:513-21.

16. Ohashi $\mathrm{Y}$, Nakai $\mathrm{Y}$, Ikeoka $\mathrm{H}$, et al. Functional and morphological pathology of the nasal mucosa after X-ray irradiation, Clin Otolaryngol 1988;13:435-46.

17. Parsons CDS, Greene CBA. A treatment for primary ciliary dyskinesia: efficacy of functional endoscopic sinus surgery. Laryngoscope 1993;103:1269-72.

18. Halma AR, Decreton S, Bijloos JM, et al. Density of epithelial cells in the normal human nose and the paranasal sinus mucosa: a scanning electron microscopic study. Rhinology 1990;28:25-32.

19. Bassiouny A, Atef AM, Raouf MA, et al. Ultrastructural ciliary changes of maxillary sinus mucosa following functional endoscopic sinus surgery: an image analysis quantitative study. J Laryngol Otol 2003;117:273-9.

20. Caciani M, Barlocco EG, Mastella G, et al. The saccharin method for testing mucociliary function in patients suspected of having primary ciliary dyskinesia. Pediatr Pulmonol 1988;5:210-4. 